\title{
Criminologie
}

\section{Show sportif et buffet froid. Interactions sportives et sociales entre détenus et joueurs extérieurs}

\section{Laurent Gras}

Volume 36, numéro 2, automne 2003

Le construit de l'ethnicité en criminologie

URI : https://id.erudit.org/iderudit/007869ar

DOI : https://doi.org/10.7202/007869ar

Aller au sommaire du numéro

\section{Éditeur(s)}

Les Presses de l'Université de Montréal

ISSN

0316-0041 (imprimé)

1492-1367 (numérique)

Découvrir la revue

Citer cet article

Gras, L. (2003). Show sportif et buffet froid. Interactions sportives et sociales entre détenus et joueurs extérieurs. Criminologie, 36(2), 105-125.

https://doi.org/10.7202/007869ar
Résumé de l'article

En France, depuis vingt ans, la dynamique d'ouverture des établissements pénitentiaires sur leur environnement s'accentue. Par l'entremise d'une offre croissante d'activités, le personnel de l'administration pénitentiaire sollicite de plus en plus de partenaires sociaux afin qu'ils participent à leur mission de réinsertion. Parmi ces activités, le sport tient une place particulière. Par sa capacité à aligner spontanément les conduites sur un langage corporel, réglementaire et rituel préétabli, la rencontre sportive entre détenus et sportifs « libres " instaure un rapport inédit entre " normaux » et " stigmatisés » quand bien même chacune des deux parties en présence appréhende quelque peu l'accueil que l'autre lui réserve. Cet événement reposant sur des bases normatives communes, il peut faire disparaître le stigmate carcéral communément attaché aux représentations de la prison et des détenus. De plus, au cours de la période d'après-match, le jeu de l'interaction sportive glisse progressivement vers celui d'une interaction sociale dans laquelle le détenu peut être reconnu dans sa dimension humaine. La rencontre sportive permet ainsi aux détenus d'apprendre à manier leur " déficience identitaire ». 


\title{
Show sportif et buffet froid. Interactions sportives et sociales entre détenus et joueurs extérieurs
}

\author{
Laurent Gras \\ Enseignant chercheur \\ École nationale d'administration pénitentiaire (ENAP) \\ Centre de recherche population et sociétés (CERPOS) \\ Université de Paris- $X$ Nanterre \\ France \\ laurent.gras@justice.fr
}

RÉSUMÉ - En France, depuis vingt ans, la dynamique d'ouverture des établissements pénitentiaires sur leur environnement s'accentue. Par l'entremise d'une offre croissante d'activités, le personnel de l'administration pénitentiaire sollicite de plus en plus de partenaires sociaux afin qu'ils participent à leur mission de réinsertion. Parmi ces activités, le sport tient une place particulière. Par sa capacité à aligner spontanément les conduites sur un langage corporel, réglementaire et rituel préétabli, la rencontre sportive entre détenus et sportifs «libres» instaure un rapport inédit entre «normaux» et «stigmatisés » quand bien même chacune des deux parties en présence appréhende quelque peu l'accueil que l'autre lui réserve. Cet événement reposant sur des bases normatives communes, il peut faire disparaître le stigmate carcéral communément attaché aux représentations de la prison et des détenus. De plus, au cours de la période d'après-match, le jeu de l'interaction sportive glisse progressivement vers celui d'une interaction sociale dans laquelle le détenu peut être reconnu dans sa dimension humaine. La rencontre sportive permet ainsi aux détenus d'apprendre à manier leur «déficience identitaire».

ABSTRACT - In France, for the last twenty years, opening up dynamics of prisons on their environment has been emphasising. By means of an increasing offer of activities, the staff of the prison authority seeks more and more social partners allowing them to participate to their mission of reintegration. Among these activities, sports hold a very particular position. Through its ability to align spontaneously the behaviours on a preestablished physical, statutory and ritual language, the match among prisoners and «free» sportsmen actually establishes an incomparable association between «regular» and «stigmatised» people even though each of them slightly dreads the greeting which the other one holds for him. This event being based on common normative foundations, it can extract the prison stigma normally linked to the representations of the 
prison and the prisoners. Furthermore, during the after match period, the sports interactions slide gradually towards that of a social interaction in which the prisoner can be recognised in his human magnitude. The match hence allows the prisoner to learn to handle its «selfdom deficiency».

Les pratiques sportives dans le milieu carcéral connaissent depuis les années 1980 un essor important résultant d'un double processus : le développement des activités physiques et sportives dans notre société et l'ouverture croissante des établissements pénitentiaires sur le monde extérieur (Soulier, 1991). Ces vingt dernières années ont ainsi vu poindre une véritable politique sportive menée par l'administration pénitentiaire aboutissant à une nette amélioration des infrastructures, une diversification des pratiques et la participation régulière d'intervenants extérieurs. Par ailleurs, les signatures d'un protocole d'accord avec le ministère de la Jeunesse et des Sports et de nombreuses conventions entre les établissements pénitentiaires et les associations et clubs sportifs locaux marquent la consécration d'un domaine d'activités jusque-là peu reconnu par les instances pénitentiaires et d'un processus d'ouverture sur le monde extérieur.

Toutefois, les effets de cette politique restent encore difficiles à apprécier : en dépit du fait qu'il soit régulièrement abordé pour illustrer les réflexions des auteurs spécialisés dans le milieu carcéral (Benguigui et al., 1992; Fabiani et Soldini, 1995; Combessie, 1996; Faure et al., 1996; Aymard et Lhuilier, 1997), le sport en prison n'a pas suffisamment fait l'objet d'investigations scientifiques pour que l'on puisse en tirer des conclusions définitives. Par ailleurs, si les résultats des seules recherches menées sur ce sujet (Courtine, 1980; Garnier et Minotti, 1993) ont permis de montrer l'aspect disciplinaire et normalisateur des activités sportives et les bienfaits plus individuels de ces dernières, la relation entre détenus et sportifs extérieurs n'a été que très succinctement abordée.

L'augmentation de compétitions sportives organisées et la multiplication des permissions de sorties sportives, autorisant le détenu à sortir de l'établissement pour participer à une manifestation sportive, posent en effet la question des relations entretenues entre les détenus et le monde libre. Plus précisément, ces rencontres sportives dévoilent, à travers des interactions produites entre détenus et joueurs extérieurs ${ }^{1}$, la problématique identitaire

1. Cet article est tiré d'une thèse de doctorat soutenue à l'Université de Paris-X Nanterre (Gras, 2001). La technique d'entretien a été la principale technique de recueil de données pour aborder notre objet d'étude. Cette approche fut essentielle pour rendre compte des représentations et des attentes des acteurs du milieu carcéral à l'égard des activités sportives. Par ailleurs, 
provoquée par l'incarcération. L'exposition publique des prestations sportives des détenus au regard d'une partie du monde extérieur sert donc l'institution carcérale, dans la mesure où elle lui permet de montrer qu'elle n'est pas aussi tyrannique que ce que l'on pourrait imaginer; mais elle sert aussi l'apprentissage du traitement carcéral et de son acceptabilité. Dès lors, il s'agit de s'interroger sur la manière dont ces rencontres sportives permettent de dépasser les effets stigmatisants dont les détenus font l'objet auprès du monde libre.

Une première hypothèse suggère que l'organisation de ce type de rencontre est facilitée par le respect des structures de l'interaction sportive. Ce type de manifestation est en effet régi par un ensemble de règles et de rituels préétablis auxquels chacune des deux parties doit répondre pour préserver l'équilibre de la rencontre. Une seconde hypothèse énonce que cet échange repose toutefois sur un équilibre précaire, normaux et stigmatisés (Goffman, 1975) devant surmonter les remparts du stigmate carcéral lorsqu'ils se trouvent face à face. Goffman (1975:25) observe en effet que

c'est lorsque les normaux et les stigmatisés viennent à se trouver matériellement en présence les uns des autres, et surtout s'ils s'efforcent de soutenir conjointement une conversation, qu'a lieu l'une des scènes primitives de la sociologie; car c'est bien souvent à ce moment-là que les deux parties se voient contraintes d'affronter directement les causes et les effets du stigmate. C'est alors que l'individu affligé d'un stigmate peut s'apercevoir qu'il ne sait pas exactement comment nous, les normaux, allons l'identifier et l'accueillir.

C'est ainsi qu'après avoir montré la manière dont les règles et les rites sportifs contribuent à l'instauration d'un échange spontané entre normaux

des questions sur les pratiques ont été posées aux détenus afin de faire émerger l'influence de ces dernières sur les représentations individuelles. Au total, 49 entretiens semi-directifs ont été réalisés, dont 21 avec les détenus, 13 avec les moniteurs de sport, 7 avec le personnel de surveillance et 4 avec les directeurs d'établissement. Parallèlement, 8 entretiens libres ont été menés avec des acteurs extérieurs aux établissements. La proportion de femmes incarcérées étant très faible ( $4 \%$ pour l'ensemble de la population carcérale et $3 \%$ en centre de détention) et leur effectif de licenciées quasi nul, aucune détenue n'a été interrogée. Mis à part cette variable, aucune caractéristique sociodémographique ni pénale n’a été retenue dans la sélectivité des détenus interrogés, si ce n'est le fait de poursuivre régulièrement une activité sportive.

Cette approche fut complétée par un travail de terrain — quatre stages d'une durée moyenne de quinze jours - dont l'objectif consistait à recueillir des documents, des lettres et des rapports, à observer les manifestations sportives et même parfois y participer. Parallèlement, 800 heures d'interventions en qualité d'animateur d'un atelier d'échecs à la maison d'arrêt de la Santé, interventions réparties sur quatre ans, ont également permis de prendre conscience des difficultés que les détenus et les moniteurs de sport des maisons d'arrêt pouvaient rencontrer dans la pratique d'un sport et la mise au point de projets. 
et stigmatisés, il s'agira ensuite de s'intéresser aux remparts du stigmate carcéral, afin de pouvoir apprécier avec plus de recul les fonctions réelles remplies par ces rencontres mixtes.

\section{L'offre de pratiques sportives}

L'image du détenu pratiquant la musculation n'est pas un mythe. Avec le football ${ }^{2}$, cette pratique physique est en effet particulièrement suivie en détention, favorisée par l'existence quasi systématique de salles de musculation au sein des établissements. Toutefois, il serait quelque peu réducteur de définir cette offre de pratique à ces deux disciplines, puisque pas moins de 30 sports sont pratiqués annuellement dans le parc pénitentiaire français (Courtine et al., 1992 : 5) 3 $^{3}$ Selon les disciplines, on constate également que l'espace des sports, ainsi que les motivations qui amènent les détenus à pratiquer, varient considérablement. Comment, effectivement, comparer l'exercice physique quotidien d'un détenu réalisé dans sa cellule avec celui réalisé dans le cadre d'une randonnée pédestre organisée en montagne?

Cette observation laisse entendre qu'ajoutées aux disciplines sportives mêmes, des «formes de pratique» viennent se greffer $^{4}$ : il s'agit des exercices physiques effectués dans les cellules, des pratiques physiques et sportives accomplies en cour de promenade et en salle de musculation, des manifestations sportives ponctuelles (invitation d'un champion, rencontre amicale contre une équipe extérieure, fête du sport...), des pratiques sportives de compétition et des permissions de sorties sportives. Avec les disciplines sportives, ces formes de pratique induisent donc des cadres de pratique spatiaux et temporels spécifiques, ainsi que divers niveaux de rencontre avec autrui. On entendra par là que pratiquer entre détenus, avec des joueurs extérieurs au sein de l'établissement ou hors de son enceinte, implique des rapports au corps et au sport, des relations et des négociations identitaires très différentes. Cette définition de notre objet d'étude nous amène ici à considérer plus en détail les enjeux engagés lors des compétitions sportives organisées entre détenus et joueurs extérieurs et les fonctions remplies par ce type de manifestation.

2. Entendu ici au sens européen du terme, équivalent au soccer nord-américain.

3. Nous citerons entre autres le tennis, le handball, le basketball, le volleyball, le badminton, le tennis de table, la boxe anglaise, le karaté, le judo, le VTT, la randonnée, le canoëkayak, le char à voile, le parachutisme...

4. Pour exemple, on comprendra aisément que le fait de marcher dans la cour de promenade n'engage pas les mêmes logiques de pratique, ni ne remplit les mêmes fonctions, que marcher sur les sentiers de randonneurs. 


\section{Les compétitions sportives}

L'instauration de pratiques sportives institutionnalisées donne la possibilité aux détenus d'adhérer à une fédération sportive nationale par la prise d'une licence et de participer à des compétitions dans le cadre d'un championnat contre des équipes ou des joueurs locaux. Ces manifestations sont donc davantage encadrées que les autres catégories de pratique et constituent l'axe prioritaire du service de sport par leur aspect éducatif et formateur. De même, elles impliquent l'adhésion des détenus à des projets d'activités et leur acceptation des moyens mis en œuvre par le référent pour y parvenir (horaire, respect, hygiène, régularité, discipline). Les sportifs faisant preuve de sérieux dans ces activités spécifiques peuvent ensuite faire l'objet de mesures d'aménagement de peine pour concrétiser tout projet élaboré avec le service. Ainsi, les pratiques sportives de compétition mettent l'accent sur l'élaboration d'objectifs à atteindre tels que le passage de grade, la montée dans des divisions supérieures et, surtout, l'apprentissage des règles officielles et des rites qui régissent la rencontre avec le monde extérieur.

\section{Règles et rites sportifs : un arrangement préétabli}

L'entrée régulière d'individus en contact direct avec la population carcérale est relativement rare dans le quotidien de la détention. Toutefois, ces rencontres sportives étant légitimées par les fonctions d'insertion qu'elles rempliraient (Rapport annuel de l'administration pénitentiaire, 2000 : 69), certains établissements prennent le risque hebdomadaire d'autoriser des joueurs extérieurs à entrer dans leurs enceintes. L'effet de ce type de manifestation et du sport en général sur l'insertion reste toutefois encore à démontrer, aucun travail n'étant parvenu jusqu'à ce jour à évaluer l'impact réel sur les comportements des détenus libérés. Fort de cette constatation, il convient donc d'appréhender le respect des règles sportives d'une manière différente de celle qu'adoptent certains spécialistes convaincus de l'effet bénéfique des pratiques sportives sur les comportements quotidiens.

Au-delà de cette approche idéalisée, les règles sportives visent avant tout à fixer les règles de l'interaction. C'est ainsi qu'à partir du moment où les détenus ont décidé de participer à des rencontres sportives contre des compétiteurs extérieurs et que, de leur côté, ces derniers ont accepté de se rendre en prison, la rencontre sportive repose sur un accord tacite consacrant un respect mutuel des deux parties. Cet équilibre instauré ne 
saurait toutefois être maintenu sans que chacun y trouve son compte. Or, les enjeux engagés dans ce type de rencontre sportive ne sont pas les mêmes pour les détenus et pour les joueurs extérieurs. Alors que les détenus useront du sport en général, et de cette rencontre en particulier, comme d'un soutien identitaire et d'un espace d'expression corporelle, les joueurs extérieurs trouveront dans cette «aventure» l'occasion de découvrir à moindre coût un pan du milieu carcéral et de vivre une expérience hors du commun. Aussi, quand bien même les premiers tenteront de prendre une revanche symbolique sur un destin difficile (Bromberger, $1998: 71)^{5}$, les seconds chercheront à éviter les heurts et à s'engager corps et âme dans le match, négociant informellement le prix de la victoire contre une rencontre loyale et le respect des règles.

\section{Le respect des règles sportives}

Les rencontres sportives présentent la particularité de permettre à deux parties qui ne se connaissent pas de parler spontanément un langage commun, corporel, réglementaire et rituel, préalablement défini. Elles permettent en cela d'instaurer d'emblée un minimum d'accords assurant le déroulement de la rencontre. Parmi ces accords, le respect des règles sportives est très probablement le plus formel. Non seulement parce que les règlements sportifs sont écrits par les plus hautes instances sportives, mais aussi en fonction de leur caractère universel, dont l'effet majeur est de parvenir à rassembler des cultures fort différentes autour de règles connues et partagées.

Toutefois, lors des rencontres sportives entre détenus et joueurs extérieurs, la signification portée à ce règlement ne consiste pas exclusivement à poser les bases légales et légitimes de l'échange sportif. Pour les reclus, le respect d'un règlement autre que celui de l'établissement auquel ils sont soumis quotidiennement leur permet de renouer avec une forme de dialogue social dont ils étaient jusqu'ici totalement exclus. Ils concevront les règles du jeu comme un soutien officiel, reconnu quasi universellement, sur lequel ils s'appuieront pour valider leurs prestations et

5. On ne manquera pas de comparer ces motivations à ce que Wacquant $(1998: 227)$ observait au sujet de la culture noire : «Dans la culture noire, le spectacle est un domaine dans lequel les noirs ont affirmé et préservé leur humanité. [...]. Cet esprit de combat et de conquête (de soi et des autres) est omniprésent dans la vie quotidienne du ghetto, qui est un univers de compétition incessante et acharnée pour la survie et le succès. La boxe est un moyen de façonner un personnage, une place protégée et une fierté rayonnante dans un monde qui menace de les condamner à l'obscurité». 
comparer leurs performances avec celles de sportifs extérieurs. Pour cela, le strict respect du règlement paraît essentiel, toutes les petites incongruités accidentelles commises par le stigmatisé pouvant être lues comme l'expression de sa différence (Goffman, 1975). L'engagement dans une rencontre sportive demande par là même aux détenus d'exprimer l'idéal sportif et de revêtir la panoplie complète du joueur fair-play plus que tout autre participant ${ }^{6}$. Garder son sang-froid, son calme et le contrôle de ses émotions devient alors un enjeu de première importance, l'occurrence de conduites déviantes faisant non seulement l'objet de sanctions prévues par le règlement sportif, mais aussi par le règlement carcéral :

Si un jour il arrive quelque chose ici, si un détenu se rebelle et qu'il frappe un adversaire, le problème c'est qu'après il n'y aura plus de championnat, je leur explique bien tout ça en début d'année (Patrice, coordonnateur de sport).

Parfois il arrive qu'on ait des altercations quant à l'arbitrage mais nous on ne peut rien dire, parce qu'on est des taulards. Si on dit quelque chose, ça peut se retourner contre nous. Si ça tourne mal, on file au mitard, c'est clair (Edouard, détenu).

D'abord sanctionné dans le cours de l'action par l'arbitre, le détenu fauteur court donc le risque de se voir doublement puni en faisant l'objet de poursuites disciplinaires. De plus, l'appartenance à l'équipe officielle de la prison représentant le plus souvent un certain prestige, une expulsion équivaudrait à une dévalorisation identitaire, cette exclusion quasi irrémédiable ne pouvant être compensée, comme à l'extérieur, par l'inscription dans un autre club. Aussi, afin de lutter contre l'apparition de tels comportements sur les terrains, les moniteurs de sport, premiers responsables du déroulement de ces rencontres, ne cessent tout au long de l'année d'avertir les joueurs des conséquences négatives, individuelles et collectives, que ce type d'actes pourrait entraîner sur la légitimité de leur

6. L'extrait suivant reste à ce titre particulièrement représentatif de cette logique : «L'institution pénitentiaire ne devait pas rater cette confrontation avec le monde libre, il lui fallait se montrer sous son meilleur jour, présenter ses meilleurs éléments dans des tenues décentes et dans un esprit sportif parfait. On acheta alors des filets neufs, les terrains furent repeints et entretenus, les détenus débouchèrent avec des aiguilles les pommes des douches réservées aux visiteurs. Le service des sports équipa ses joueurs de maillots derniers cris, il acheta des ballons réglementaires et repeignit le tableau d'affichage. Désormais, tous les dimanche matins, la prison ouvrait ses portes et se mettait sur son « 31 » pour montrer à ses visiteurs qu'elle n'était pas si dure qu'on le disait à l'extérieur. Le personnel d'encadrement voyait sa tâche singulièrement revalorisée, il lui fallait produire une équipe solide, efficace, correcte; il lui fallait montrer que les exclus pouvaient faire preuve de toutes les qualités des hommes libres. Le climat de ces rencontres fut exceptionnel, chacun en rajoutait pour montrer son «fair-play», les chocs étaient suivis de poignées de main amicales...» (Courtine, 1980 : 176-177). 
participation. Pour éviter que les équipes extérieures ne s'essoufflent du fait de comportements irrespectueux et des multiples contraintes dues à leur déplacement ${ }^{7}$, on comprend dès lors l'importance de l'apprentissage du respect des règles et de l'adversaire, principaux garants de la pérennisation de telles manifestations :

Parce que pour tenir un championnat dans une prison et par rapport aux équipes extérieures qui ont déjà une certaine réticence à l'idée de rentrer dans une prison, ils ne savent pas ce qui les attend, il faut que ça se passe bien, le fair-play total. Si les gens viennent ici pour se faire menacer, insulter ou prendre des coups, c'est fini (Daniel, moniteur de sport).

Pour les joueurs extérieurs, cet arrangement réglementaire prédéterminé apparaîtra comme une forme de sécurité à laquelle ils pourront se référer pour maintenir l'ordre de l'interaction et évacuer en partie certaines appréhensions naturelles. Bien qu'ils ne soient pas conscients des sanctions carcérales dont les détenus pourraient faire l'objet en cas d'incidents, ces intervenants savent pertinemment que la règle sportive est une caution suffisamment fiable pour garantir la correction et le bon déroulement de la rencontre. Ces règles de l'échange physique — matérialisées par un mode de comportement particulier que les participants doivent suivre, par des sanctions encourues pour des fautes de jeu, par des rôles spécifiques à jouer - leur permettent en effet de régler leur propre conduite tout en anticipant celle de leurs adversaires. Ces lignes de jeu formelles ne sont toutefois pas les seules à assurer la qualité de l'échange, le respect des rites sportifs étant également essentiel.

\section{Le respect des rites sportifs}

L'ordre de l'interaction sportive est aussi maintenu par une série de rituels donnant au sport une dimension cérémonielle (Goffman, 1968 : 157159 ; Bromberger, 1995 : 319-346). Définie à l'origine autour d'attributs et de rangs précis à tenir par rapport à l'objet de la célébration, la fonction du rituel consiste en effet à favoriser l'émergence «d'une communion des consciences» et d'un accord global autour de valeurs essentielles, de «s'attester à soi-même et d'attester à autrui qu'on fait partie d'un même groupe» (Durkheim, 1990). Multipliant les analogies entre l'organisation

7. Fouilles, vérifications des identités avant et après les rencontres, enquête, risques de ne pas franchir l'enceinte pour une raison $x$, appel, contre appel, sont le lot commun de toutes les équipes sportives entrant dans un établissement pénitentiaire. 
des matchs de football et celles des manifestations religieuses, Bromberger montre ainsi la manière dont les rencontres sportives sont empreintes d'une dimension rituelle. Parmi les rituels observés, la dimension cyclique du calendrier des rencontres, la maîtrise du code technique distinguant l'initié du profane, l'universalité de la réglementation et des lois sportives, le découpage territorial des instances fédérales et les particularismes locaux (qui ne sont pas sans rappeler les relations complexes entretenues entre religion officielle et religion populaire) indiquent combien les rencontres sportives établissent une véritable communion des consciences où disparaissent momentanément toutes hiérarchies ordinaires dans une effervescence collective ${ }^{8}$. Pour un détenu,

Le sport en prison porte une certaine unité, une communion, une humilité. Le sport réunit des individus qui ont parfois du mal à trouver une place, à se situer dans ce milieu particulier sous un discours commun, un thème central. C'est une chose à partager. Le sport rassemble des individus aux trajectoires différentes, ils sont différents en âge aussi, les classes sociales auxquelles ils appartiennent sont parfois éloignées. Le sport unifie tout cela dans un univers avilissant (Alain, détenu).

Transcendant les barrières des structures sociales, explicitées dans ce témoignage par les rapports entre générations, entre classes sociales mais aussi entre reclus et le monde extérieur, la rencontre sportive est ici présentée comme une forme de communion solennelle, une rupture avec le quotidien carcéral, une expérience singulière contribuant à pondérer les différences culturelles des groupes d'appartenance respectifs :

Les équipes adverses savent que nous sommes des détenus mais des joueurs de handball aussi donc à partir du moment où le coup de sifflet retentit, le match commence et les barrières sautent... (Vincent, détenu).

La rencontre sportive marque consécutivement un niveau de croyance élevé. Il est un moment sacré, quasiment une union mystique où le travail de figuration doit être sauvegardé au risque de faire courir un danger immédiat à l'équilibre de la relation sportive. À cette fin, cette coopération dramaturgique, traduite par le revêtement du masque sportif, est empreinte

8. Christian Bromberger ajoute aussi que la sacralisation du stade par les supporters, les pèlerinages religieux organisés par certains groupes sportifs, l'analogie structurelle de la répartition du public dans le stade avec la distribution précise des groupes sociaux lors des cérémonies religieuses, le regroupement des fidèles sportifs en confréries, l'existence de retraites organisées par les joueurs avant les grands matchs dressent une véritable effervescence émotionnelle, une telle densité symbolique des valeurs mises en jeu, un tel drame sacrificiel, que l'on ne peut que prendre au sérieux le parallèle établi entre le match de football et le rituel religieux (Bromberger, 1995 : 311-346). 
de vénérations multiples qui font que le social tient (Goffman, 1993 : $43)^{9}$. Ces rites sportifs sont très nombreux. À titre d'exemple, pour exprimer l'esprit de fair-play dans lequel deux boxeurs abordent un combat, il est d'usage de les voir se taper les gants, sous-entendant ainsi leur volonté de mener le combat dans les règles de l'art, sans coup bas. À la fin du combat, il est également courant que les deux rivaux s'étreignent, laissant entendre que l'agressivité qu'ils ont exprimée à l'encontre de l'autre n'était qu'un emportement de circonstance ne dépassant aucunement les limites temporelles et spatiales de la compétition. De même, lors des compétitions de sport collectif, il est fréquent qu'une équipe envoie volontairement la balle en touche pour qu'une équipe de soins entre sur le terrain afin de s'occuper de la blessure d'un joueur adverse que l'arbitre n'a pas remarquée. En signe de reconnaissance, l'équipe adverse prendra à son tour le soin de rendre le ballon dont elle n'avait pas la possession avant l'interruption de la séquence de jeu. Dans le cas où les coéquipiers du joueur blessé ne réagiraient pas de cette manière, l'équilibre de l'interaction sportive risquerait alors de disparaitre et de laisser place à des déconvenues pouvant sérieusement perturber le déroulement de la rencontre, parfois plus encore que si un point précis du règlement eût été enfreint :

C'est sûr, l'arbitre, il ne voit pas toujours tout. Tous les joueurs de foot le disent : il y a le règlement et tous les à-côtés qui peuvent être des accolades entre joueurs, l'un aide l'autre à se relever, comme des rapports un peu plus tendus, des regards, des gestes qui en disent parfois plus qu'un long discours. C'est tous ces petits gestes qui permettent le bon déroulement du match. C'est aussi une marque de respect, une manière d'agir et de se comporter loyalement (Pierre, détenu).

Le partage des croyances et de rites de pratique lors d'événements sportifs peut à ce titre être pleinement considéré comme un régulateur de tensions aussi efficace que le respect des règles. En raison de sa forte symbolique, il est une preuve réelle de l'état d'esprit dans lequel les participants s'engagent dans la partie. Par ailleurs, la considération portée à ces rites permet de détourner l'attention furtive que chacun serait susceptible de porter aux éventuels effets du stigmate durant la rencontre et de s'engager entièrement dans le contenu officiel de l'interaction. À l'inverse, son irrespect souligne immédiatement la précarité de cet équilibre, d'autant plus que l'une des parties est soupçonnée d'avoir peu, sinon

9. Ramenant ainsi les pratiques sociales constitutives de l'intégrité et de la solidarité des groupes à l'échelle des personnalités individuelles et de l'accomplissement d'actes symboliques réciproques faisant de la personne un être sacré. 
aucune moralité. L'observation de ces rites sportifs reste à ce titre particulièrement déterminante dans le processus de désignation sportive et pour la préservation de l'ordre de l'interaction ${ }^{10}$. On ne saurait donc manquer ici de signaler la dimension religieuse des règles et des rites sportifs auxquels chacune des parties obéit, le langage sportif recouvrant tellement d'autorité qu'à l'instar de toute cérémonie religieuse, la moindre dérogation pourrait être perçue comme un véritable sacrilège.

Répartie sur l'année, l'organisation de manifestations sportives multiplie donc les occasions pour les détenus de partager une croyance commune avec le monde extérieur, confondant sur une même scène normaux et stigmatisés. La répétition cyclique des rencontres sportives est un élément essentiel pour que de telles célébrations collectives puissent agir comme des «désidentificateurs» (Goffman, $1975: 60)^{11}$ en permettant aux détenus de faire bonne figure. Car si la singularité de ces «contacts mixtes» permet effectivement d'assurer dès la première rencontre l'ordre de l'interaction, il n'en reste pas moins qu'en fond de toile de ce langage préétabli demeure une appréhension latente relative à l'identité stigmatisante des détenus, ainsi qu'à leurs difficultés à surmonter les remparts de leurs déficiences pour s'affirmer en tant que sportif et, plus largement, en tant qu'homme.

\section{Les remparts du stigmate carcéral}

Goffman définit le stigmate comme un attribut qui jette un discrédit profond dans une relation (Goffman, 1975 : 13). Une des propriétés majeures des stigmates réside dans leur degré de visibilité, chacun d'entre eux n'étant pas immédiatement repérable. Pour marquer cette distinction, Goffman définit deux sortes de stigmates : ceux qui ne peuvent être aisément dissimulés (difformités physiques, couleur de peau, déficience verbale) et ceux qui peuvent être gardés secrets (alcoolisme, maladie mentale, travers physique ou mental, déficience physiologique). Si la possession du premier type de stigmate fait de ceux qui les portent des êtres discrédités, celle d'un stigmate non perceptible rend l'individu discréditable. Cette dimension de visibilité est d'autant plus importante que

10. Il fut d'ailleurs fréquent d'entendre les moniteurs de sport se réjouir d'avoir obtenu la coupe du fair-play et exprimer leur satisfaction quant au bon déroulement des rencontres.

11. Goffman emploie ce terme pour définir les signes qui tendent, en réalité ou en espérance, à briser les représentations stigmatisantes convenues et préétablies d'un individu porteur d'une tare. 
la découverte d'un stigmate induit pour autrui un réajustement général de la conception identitaire établie jusqu'alors, chacune des conduites menées par le stigmatisé n'étant plus interprétée qu' à travers sa différence.

Contrairement aux permissions de sorties sportives où les détenus, mêlés aux sportifs du monde extérieur, ne sont pas toujours reconnus en tant que tel, les rencontres sportives qui se déroulent dans l'enceinte pénitentiaire présentent la particularité d'identifier immédiatement les détenus, de mettre en avant le profond discrédit dû à l'incarcération et d'en mettre au jour les effets. Discrédités, le problème qui se pose à eux consiste alors à adopter au plus près le comportement $\mathrm{du}$ «bon sportif», afin de se détacher des représentations stigmatisantes que les joueurs possèdent à leur égard. L'arrangement relatif à la rencontre ne résulte donc pas uniquement du cadre réglementaire et rituel de l'événement. Il dépend aussi de son traitement par les hommes.

Parmi les acteurs chargés d'atténuer cet effet négatif, les moniteurs de sport, organisateurs de ces rencontres, sont les premiers responsables. Cette mission est dans un premier temps assurée au cours des entretiens téléphoniques menés avec les dirigeants sportifs extérieurs avant les rencontres. Si ces communications ont pour objectif majeur de poser les bases organisationnelles de la rencontre, elles visent plus informellement à rassurer les dirigeants extérieurs quant à l'accueil qui leur sera réservé :

C'est pas évident de tenir une équipe en championnat ici, on a déjà eu pas mal de petits incidents, bon c'est vrai qu'à partir du moment où les équipes viennent ici, elles perdent pas mal de leurs moyens. Quand on arrive dans un $\mathrm{CD}$, on ne sait pas sur qui on va tomber, comment ça va se passer. Alors quand j'ai les dirigeants au téléphone, j'essaie au mieux de les mettre à l'aise en leur disant par exemple que je serai présent dès leur entrée jusqu'à leur sortie (Dominique, moniteur de sport).

Conscient de ces appréhensions, le moniteur de sport sait consécutivement que l'accueil sportif (vestaires, douches, arbitrage...) qu'il réserve aux dirigeants et aux joueurs extérieurs doit non seulement s'aligner sur le modèle extérieur, mais aussi réserver l'assurance d'un encadrement parfait, parfois agrémenté d'une copieuse troisième mi-temps :

Tous les quinze jours, des boxeurs extérieurs entrent. Lors des grandes réunions de boxe, tout un cérémonial, présentation officielle, enregistrement vidéo, buffet froid avec les pratiquants de l'extérieur au mess, petits pots avec les détenus. On s'engage à bien les recevoir (Yves, moniteur de sport).

Moi, $j$ 'ai toujours dit que les gars, ils ne viennent pas ici pour se faire insulter. Je préviens mes joueurs et je fais toujours en sorte de bien recevoir les 
joueurs qui viennent de loin en plus, parfois. On a un meuble ici, rempli de boissons, cocas, jus de fruits, qu'on sort en fin de match. Il arrive aussi que des détenus se cotisent ou préparent des tartes ou des gâteaux (Daniel, moniteur de sport).

Pour les détenus, ces contacts mixtes suscitent des interrogations quant aux réactions de rejet ou d'acceptation tant que le premier contact n'est pas pris. En raison de leur appartenance à la sélection et parce que l'institution leur reconnaît les capacités de pouvoir se confronter aux compétiteurs extérieurs, les détenus se voient investis d'un nouveau rôle consistant à porter et à honorer les couleurs de la population carcérale. Ils savent donc qu'ils auront tout intérêt à fournir une bonne prestation et à contrôler l'impression qu'ils vont produire. Dans l'équipe, censée être composée des meilleurs joueurs, «les vedettes assument [donc] un rôle qui déborde largement l'image stéréotypée que l'on se fait habituellement du reclus, puisque le sport d'équipe exige des qualités telles que l'intelligence, l'adresse, l'endurance, l'esprit d'équipe et même le sens de l'honneur» (Goffman, $1968: 158)$.

L'incertitude plane également chez les visiteurs quant à la qualité de l'accueil que leur réserve une population dont ils n'ont que des représentations biaisées, voire quelque peu caricaturales. Aussi, à l'approche du jour de match, des murs de la prison, de la porte d'entrée, des nombreuses grilles et des vestiaires, le fait même de disputer une rencontre sportive contre des détenus les interpelle de plus en plus sur la manière dont ils vont jouer : irrespectueusement, peut-être même y aura-t-il des insultes, des bagarres. C'est ainsi que pour les joueurs extérieurs, l'identification première des détenus tient principalement à leur identité de délinquants, et non pas à celle de sportifs :

Oui, ce que je trouve dommage, c'est que c'est toujours négatif. Les gens de l'extérieur ne connaissent pas l'intérieur. Sur quoi ils se basent? Sur la télévision? Ils voient un film américain, avec des gros balèzes et, en fin de compte, ils s'aperçoivent que c'est des gens comme tout le monde (Marc, coordonnateur).

La première fois on est toujours impressionné et quand ils en sortent ils sont contents d'avoir vécu cette expérience. J'avais une image de la prison un peu ancienne, les cachots. La prison en fait on se l'imagine surtout au niveau des cellules, des gros méchants qui peuvent y habiter (Antoine, joueur extérieur).

L'avant-match génère pour ces raisons des angoisses et des peurs que tout premier entrant a plus ou moins éprouvées en franchissant le seuil de ces enceintes. L'appréhension demeure à ce titre un sentiment partagé par 
l'ensemble des participants extérieurs et qui est fortement ressentie par les détenus :

Les joueurs, arbitres, physiquement ça se voyait qu'ils n'étaient pas à l'aise, ils étaient marqués (Antonio, détenu).

Ils avaient peur de venir jouer ici, je ne sais pas comment le dire mais ça se voyait dans leur manière de jouer, dans leur engagement physique. Je suis habitué à voir des gars jouer au foot, il n'y avait pas ce contact qu'on voit sur les stades, ils ne se livraient pas de la même manière. Alors avec le public qui criait autour... (Daniel, moniteur de sport).

Parce qu'ils sont conscients du fait que ce type de situation ne présente rien d'ordinaire, les joueurs extérieurs cherchent fréquemment à dissimuler leur malaise. Il est ainsi courant d'observer qu'une fois la première porte passée, quelques plaisanteries sont lancées afin de détendre l'atmosphère. Les blagues les plus répandues consistent généralement à prévenir un compagnon des risques qu'il court d'être reconnu et de rester enfermé ou encore du danger qu'il court à cacher sous ses vêtements de sport la traditionnelle lime à scier les barreaux.

D'autres se reprochent d'avoir omis d'apporter des oranges. Effectivement, l'expression était toute trouvée tant on sait l'importance de faire le plein de vitamines avant de disputer une compétition sportive. Enfin, parce que leurs proches ressentent de l'appréhension, certains dissimulent cette aventure aux membres de leur famille pour ne pas les effrayer. Un arbitre fédéral confia ainsi qu'il avait caché à sa femme les rencontres qu'il était venu arbitrer au centre de détention, laissant entendre que celle-ci aurait pu exprimer quelques réticences à ce qu'il s'y rende.

Globalement, ces quelques observations laissent donc entrevoir l'existence d'une incertitude latente qui, annihilée en partie par les cadres réglementaire et rituel de la rencontre, resurgit lorsque normaux et stigmatisés se rencontrent hors du terrain sportif.

\section{Les clefs de la troisième mi-temps}

Dans certains centres de détention, l'entrée d'équipes extérieures remonte à plusieurs années. Ayant pris conscience que les détenus pouvaient respecter les règles et suivre les mêmes rituels sportifs, les joueurs extérieurs comprennent que les conduites imaginées des détenus ne correspondent pas à celles observées. Toutefois, cet alignement des conduites sportives paraîtrait quelque peu limité s'il n'était poursuivi par aucun autre type de 
communication, l'objectif de ces manifestations consistant également à amener les deux parties à partager une certaine sociabilité.

Un des moments privilégiés investi pour l'entretien de tels échanges est la «troisième mi-temps» que les joueurs des deux parties mettent à profit pour discuter et partager une certaine convivialité. Les organisateurs supposent en effet que l'échange physique, l'entente réglementaire et rituelle de la rencontre sportive ayant permis de montrer aux deux parties qu'un accord pouvait être possible, l'amorce d'une discussion entre normaux et stigmatisés s'en retrouve facilitée. L'équilibre du déroulement de cette période d'après match repose donc implicitement sur cette entente préalable sans laquelle l'échange trouverait probablement quelques difficultés à s'établir. Pourtant, si le prétexte sportif parait idéal pour réaliser cette mise en situation, ces moments sont parfois loin de répondre aux attentes projetées. Ce «face-à-face collectif», où les deux parties se trouvent en situation de discuter et de mener des conversations, révèle en effet une structure d'interaction que les joueurs ne sont pas toujours à même de maîtriser. Le fait même de placer en face-à-face normaux et stigmatisés peut consécutivement sembler problématique dans la mesure où, le langage sportif rompu, tous ne présentent pas les mêmes dispositions à nouer contact et surtout à pouvoir apprécier les sujets de conversation qu'il est possible d'aborder. Dès lors, quand bien même cette troisième mi-temps avait été pensée dans le prolongement d'un échange partagé après lequel l'amorce d'une discussion devait théoriquement s'effectuer, l'observation de son déroulement laisse davantage poindre la réalité d'une situation problématique non plus réglée par le partage d'un langage sportif préétabli, mais par les tentatives réciproques de chaque partie de considérer l'autre sans commettre d'impairs.

Les détenus recevant, certains d'entre eux comprennent alors qu'il leur convient d'adopter des comportements d'accueil et de service à l'égard des visiteurs. Parmi les moyens mis en œuvre pour apaiser cette tension latente, certains détenus ont la charge de servir les verres et de proposer les quelques mets préparés ou commandés pour la circonstance. Par ailleurs, afin de surmonter le froid qui caractérise ces situations, les détenus essaient de détendre leurs interlocuteurs en émettant quelques paroles rassurantes sur la nature de leurs intentions :

On les mettait à l'aise. Bon les mecs ils rentrent pour la première fois, ils sont un peu effrayés, je le voyais, les arbitres à la fin du match c'est moi qui les ramenais jusqu'à la porte de la prison (Nicolas, détenu). 
On le voit quand ils sont en retrait, ou même à la fin du match, on fait un pot de l'amitié, tout le monde se retrouve dans notre vestiaire, on en voit qui restent dans leur coin ou entre eux qui discutent. Moi, avec quelques autres, on essaie en passant par le biais du foot de décoincer un peu (Eric, détenu).

Quand ils viennent en prison ils ont une appréhension, ils n'osent pas trop... même par la parole. Dans ce cas là, c'est à nous d'aller vers eux, de faire la démarche. Eux font la démarche de venir, c'est à nous de les accueillir, de les recevoir. C'est l'image du détenu, le mec regarde les médias, à travers ce qu'on en dit... en fin de compte on a fait une connerie, on n'est pas différent, on n'est pas des sauvages. Le mec ne va pas se faire agresser, mais ça c'est à nous de le montrer de suite, «comment tu t'appelles, machin...», et de fil en aiguille, il se dit «je n'ai pas un groupe de sauvages en face de moi» (Patrick, détenu).

Les rencontres mixtes offrent donc aux détenus l'opportunité d'apprendre les structures de l'interaction et d'en gérer les mécanismes risquant d'interférer par le poids de leur stigmate. De fait, ce type de situation permet aux détenus de découvrir et de recomposer des lignes de conduite auxquelles ils doivent obéir afin d'aider les autres à s'engager dans le contenu officiel d'une conversation et de minimiser l'importunité de leur déficience. On comprendra par là que les contacts mixtes répétés auxquels les détenus participent leur permettent d'apprendre la façon de traiter les normaux et de les aider grâce au savoir-faire qu'ils ont acquis au fil des rencontres (Goffman, 1975 : 137-139).

Cet apprentissage n'est cependant pas univoque, le comportement des joueurs extérieurs connaissant à leur tour une certaine évolution :

C'est sûr, au début, on n'était pas trop rassuré. Puis, avec les années, on a appris à connaître les gars et à parler avec eux. C'est là qu'on s'est aperçu qu'on pouvait parler normalement avec eux comme on parle tous les jours au voisin, au collègue de bureau (Eric, joueur extérieur).

Constatant qu'il était possible de parler «normalement» avec un détenu, ce joueur laisse entendre que les représentations qu'ils portaient initialement à l'égard de la population carcérale présentaient des attributs ne pouvant pas convenir à l'entretien d'un échange ordinaire. En précisant ensuite que cette conversation pourrait tout à fait être menée avec divers protagonistes de la vie courante, cet interlocuteur associe le détenu à une personne de la vie quotidienne, parfaitement capable de jouer un rôle social structuré et standardisé. Au cours de ce processus, on voit donc ses représentations évoluer. D'abord porteurs de visions stéréotypées, les visiteurs découvrent ensuite des individus capables de disputer 
une rencontre sportive dans les règles, puis de tenir une conversation sans que celle-ci ne soit trop entravée par leur situation stigmatisante. $\mathrm{Au}$ cours de ce parcours que l'on pourrait qualifier d'initiatique ${ }^{12}$, les joueurs extérieurs voient au fil du temps se resserrer les relations qu' ils ont avec les détenus et leurs premières réactions stéréotypées disparaitre progressivement. Peu à peu, le froid des premières rencontres laisse alors la place à une certaine sympathie et à la compréhension où l'appartenance stigmatisante des uns cesse d'être un facteur crucial et dérangeant pour le maintien de l'interaction :

C'est plus évolué maintenant. Peut-être parce que les équipes ont l'habitude de venir et puis ce sont des équipes qu'on connaît qui reviennent, toujours les mêmes en championnat. Tu vois qu'il y a une recherche, ce n'est pas pour le zoo, car ils ne te parlent pas de la prison (Frédéric, détenu).

Après, ces gens là revenaient, ils avaient une autre approche. Ils étaient beaucoup plus libres, ils discutaient un peu de tout, hors prison. Au début, ils étaient d'un côté , nous un peu de l'autre (Didier, détenu).

Toutefois, cette évolution ne se réalise pas d'une manière anarchique, chacun des protagonistes évitant d'aborder l'objet de la déficience. Mode de figuration spécifique que Goffman rattache à la diplomatie et au tact employés pour éviter que l'autre ne perde la face (Goffman, 1974 : 16), la fonction remplie par ce procédé d'évitement vise à écarter les sujets et les activités qui pourraient déséquilibrer la conversation en instaurant un malaise d'autant plus important que l'échange sportif est censé valoriser le détenu plus que de le mettre à mal. L'ignorance volontaire de ce que le détenu dissimule expressément permet ainsi au visiteur de répondre aux attentes de son interlocuteur et de lui donner l'opportunité de faire bonne figure. Ce ménagement des faces n'implique toutefois aucunement le fait que le joueur extérieur soit totalement désintéressé de connaitre le passé du détenu. Pour cela, il devra attendre qu'une certaine confiance s'installe et user de grandes précautions avant d'aborder le sujet ${ }^{13}$. Bien que l'étude de terrain n'ait pas permis de dresser une analyse approfondie

12. Car entrer ne serait-ce qu'une fois en prison, c'est aussi apprendre à intérioriser la hauteur des murs, des nombreuses portes et grilles qui se referment derrière nous, parfois aussi la salubrité et l'étroitesse de l'environnement, les multiples regards de surveillants et de détenus, ou encore les sonorités particulières composées de bruits de clés et de cris déchirant l'espace.

13. Il peut arriver que des liens et des affinités plus personnelles se nouent par l'entremise des rencontres sportives répétées. C'est ainsi qu'un détenu évoqua les contacts réguliers qu'il avait instaurés avec des joueurs extérieurs qui lui avaient rendu de grands services en lui procurant des ouvrages sportifs et en se démenant pour lui permettre de s'inscrire à des cours par correspondance. 
des discussions et des modes de figuration utilisés au cours de ces contacts mixtes, dirigeants et entraîneurs extérieurs sont globalement, aux dires des moniteurs de sport, les premiers à s'engager à poser des questions sur les antécédents des détenus. Ce constat provient probablement du fait que par leur statut, ils se voient conférer la légitimité de demander des informations plus fouillées sur les coulisses de la scène carcérale. On comprendra par là même que la tolérance dont ils font preuve en acceptant de participer à une compétition dans une prison exige bien en retour quelques compensations informatives :

Parfois, les extérieurs disent «tiens, ici qu'est-ce que vous avez, un peu de tout, combien ils ont à faire? » Bien sûr je ne leur dis pas, secret professionnel. Les gars, ils le savent c'est plus gênant de dire «tiens celui-là que tu as dans l'équipe qu'est-ce qu'il a fait?» que de dire «tiens ceux de ton équipe en général qu'est-ce qu'ils ont fait, ils sont là pour longtemps?». Oui, ça arrive fréquemment ce genre de questions. Quand l'entraîneur demande des précisions individuelles, c'était arrivé lors d'un match, «tiens celui qui a des tresses, il joue pas mal», alors je dis que moi ça ne me regarde pas et que je ne sais pas, ou encore que je ne peux pas leur dire. Ils le remarquent à un certain comportement ou au «look», tiens celui qui a les grands cheveux qu'estce qu'il a fait, ou alors parce qu'effectivement il joue bien, «tiens qu'est-ce qu'il a fait ton joueur pour en arriver là, il jouait avant?» (Daniel, détenu).

Afin d'éviter de s'adresser directement aux détenus et de se heurter aux obstacles évoqués plus haut, les responsables de l'équipe extérieure sollicitent les moniteurs de sport au nom de leurs positions respectives communes. Conscients du fait que les révélations qu'ils ambitionnent de connaître sont susceptibles de relever d'une curiosité mal placée qui pourrait être interprétée négativement, les dirigeants des clubs locaux essaient de justifier leur démarche et d'introduire leur requête en évoquant un attribut physique, comportemental ou sportif particulier présenté par l'individu dont il est question. Cette preuve de tact montre que l'utilisation de telles méthodes n'est donc pas exclusivement réservée aux contacts directs entretenus avec le stigmatisé mais également appliquée à son entourage. Évoquant le secret professionnel pour ne pas divulguer ce genre d'information, le moniteur de sport remplit le rôle du médiateur institutionnel et prend d'une certaine manière le rôle du détenu en réactivant à son tour certaines stratégies de dissimulation. De même, en faisant observer à son interlocuteur qu'il ne connaît pas lui-même la nature des contentieux et que cela ne le regarde pas, il laisse entendre subtilement que l'intérêt porté à ce type de question n'est pas d'actualité. En 
outre, ce type de réaction montre également la capacité du moniteur de sport à protéger l'identité stigmatisante des détenus et à contrôler la circulation d'informations pouvant déprécier leur image.

De façon plus générale, il est donc intéressant d'observer que les rencontres sportives mixtes visent tout autant l'instauration d'un contact entre normaux et stigmatisés que l'obtention d'un résultat sportif. L'objectif réel de ces rencontres réside également dans les tensions qu'elles génèrent et l'apprentissage subreptice des rites d'interaction que les deux parties doivent apprendre à contrôler. Si la troisième mi-temps est habituellement vouée à la fête et aux débordements de toutes sortes, celle ayant cours entre détenus et visiteurs reste davantage marquée par la gestion d'une tension latente et d'un équilibre précaire. Au cours de ces interactions répétées, détenus et visiteurs apprendront alors à manier avec plus d'aisance l'objet du malaise. Les premiers trouveront au sein de ces situations une occasion privilégiée d'apprendre à manier leur stigmate, les seconds, l'opportunité de faire évoluer leurs représentations dans un parcours initiatique. En cela, on peut suggérer l'idée selon laquelle le sport en prison remplit une fonction préventive en apprenant aux stigmatisés et aux normaux les structures de l'interaction qui règlent les lignes de conduites réciproques lorsque ces deux univers se rencontrent.

\section{Conclusion}

Le champ carcéral demeure un lieu difficile d'accès quand bien même l'administration pénitentiaire autorise de plus en plus de chercheurs à l'investir pour mener leurs investigations. L'impératif de confidentialité, généré par les contraintes sécuritaires, ne permet pas, en effet, de formuler toutes les informations en n'importe quelles circonstances. De fait, ces résistances induisent une complexité certaine, érigeant pour l'analyse sociologique un certain nombre d'obstacles. Aussi, à l'instar de Hughes (1996 : 30-31) préconisant «l'examen de cas offrant la moindre résistance à l'analyse sociologique» pour tirer de l'information sur un milieu sensible, l'intérêt porté au sport, pratique sociale jugée futile ${ }^{14}$, recouvre un caractère fécond pour aborder des questions de fond posées par le rapport des individus à l'institution carcérale et, par extension, interroger, à

14. À titre d'exemple, Duret (2001:5) souligne combien «l'objet sport» «est souvent considéré comme un phénomène négligeable ou insignifiant»; dans une perspective identique, Bromberger (1995: 314) perçoit le sport comme un objet d'étude nécessitant «de fortes prothèses pour acquérir quelques dignités dans le champ de la connaissance». 
travers le «prisme sportif», les difficultés d'ouverture de l'administration pénitentiaire sur le monde extérieur.

Trop souvent présentées comme un moyen de réinsertion sans qu'aucune étude scientifique ne soit à même de rendre compte de l'impact de cette politique sportive sur les trajectoires sociales suivies après la libération, les activités sportives en prison présentent toutefois l'intérêt de remplir d'autres types de fonction. Outre la confrontation sportive en elle-même et l'oxygénation physique et relationnelle procurée aux détenus, l'observation de ces manifestations permet en effet de constater la manière dont ce show sportif favorise l'amorce d'une relation entre normaux et stigmatisés, donnant à ces derniers l'opportunité de sortir de leur condition de reclus. Réciproquement, la confrontation directe de citoyens ordinaires avec un pan du milieu carcéral offre un espace-temps au sein et au cours duquel les a priori négatifs liés à l'identité du détenu sont remis en cause. Ce processus ne s'établit toutefois pas spontanément. Ce type de manifestation n'induit effectivement pas l'instauration d'un échange aussi entendu que celui observé dans l'échange sportif. L'intérêt de telles rencontres consiste alors à faire émerger les écueils relationnels qui se posent lorsque chacune des deux parties se retrouve en face à face lors de la troisième mi-temps autour d'un buffet froid. S'opère alors un glissement progressif qui amène non plus à traiter de la scène sportive mais plutôt des interactions produites entre stigmatisés et normaux dans le cadre d'une situation ordinaire. Parce qu'à ce moment précis, le sport contribue à dévoiler les effets stigmatisants de l'incarcération, il remplit une fonction préventive en permettant aux détenus de prendre conscience de leur différence et d'adopter des lignes de conduite adaptées pour la surmonter. Par ailleurs, s'il paraît relativement complexe de démontrer que l'apprentissage de l'habilité à négocier une identité plus valorisante dans ce contexte favorise le traitement personnel de l'identité d'ex-détenu après la libération, l'évolution du regard porté par les joueurs extérieurs sur la prison et sur les détenus sous-entend l'idée selon laquelle les représentations ne sont pas figées mais varient dans le temps. Ce n'est donc pas tant l'expérimentation de conduites en ellesmêmes, variables selon les circonstances, qu'il convient de retenir, que la dynamique qui les anime. Pour cette raison, constater que les normaux découvrent avec le temps la dimension humaine de ceux qu'ils pensaient en être dépourvus permet d'envisager l'idée d'un possible changement des considérations stéréotypées portées sur l'identité d'ex-détenu. 


\section{Références}

Aymard, N., \& Lhuilier, D. (1997). L'univers pénitentiaire, du côté des surveillants de prison. Paris : Desclée de Brouwer.

Bromberger, C. (1995). Le match de football, ethnologie d'une passion partisane à Marseille, Naples et Turin. Paris : Ethnologie de la France, La maison des sciences de l'homme.

Bromberger, C. (1998). Football, la bagatelle la plus sérieuse du monde. Paris : Bayard.

Chauvenet, A., Benguigui, G., \& Orlic, F. (1992). Le personnel de surveillance des prisons. Essai de sociologie du travail. Centre d'Etude des Mouvements Sociaux, CNRS.

Combessie, P. (1996). Prisons des villes et prisons des campagnes, étude d'écologie sociale. Paris : Les éditions de l'Atelier.

Courtine, F. (1980). La sportification pénitentiaire : de la "roue au ballon... prisonnier». Thèse de doctorat, UER de sociologie, Université de Paris VII.

Courtine, F., Fillet, B., \& Siret, R. (1992). Rapport du groupe de travail pour l'actualisation des instructions relatives aux activités physiques et sportives en établissement pénitentiaire. Paris : Ministère de la Justice.

Duret, P., \& Trabal, P. ( 2001). Le sport et ses affaires, une sociologie de la justice de l'épreuve sportive. Paris : Métailié

Durkheim, E. (1990). Les formes élémentaires de la vie religieuse. Paris : PUF.

Fabiani, J.-L., \& Soldini, F. (1995). Lire en prison, une étude sociologique. Paris : Études et Recherches, Ministère de la Culture.

Faure M., Mathieu, L., \& Welzer-Lang, D. (1996). Sexualités et violences en prison. Observatoire Internationale des Prisons, Lyon : Aléas éditeur.

Garnier, S., \& Minotti, C. (1993). Influence des activités physiques et sportives sur les conduites de santé des fermmes incarcérées. Thèse de STAPS, Université de Nancy I.

Goffman, E. (1968). Asiles. Études sur la condition sociale des malades mentaux. Paris : Minuit.

Goffman, E. (1974). Les rites d'interaction. Paris : Minuit.

Goffman, E. (1975). Stigmate. Les usages sociaux des handicaps. Paris : Minuit.

Goffman, E. (1993). Les rites d'interaction. Paris : Minuit.

Gras, L. (2001). Le sport en prison, analyse socio-démographique des carrières sportives de détenus. Thèse de socio-démographie, Université de Paris-X Nanterre.

Hughes, E. (1996). Le regard sociologique, essais choisis. Paris : Éditions de l'EHESS.

Ministère de la Justice (2000). Rapport annuel de l'Administration Pénitentiaire. Ministère de la Justice, France.

Soulier, G. (1991). Sur une perspective de transformation de la prison : décloisonnement ou désinstitutionnalisation. Revue de droit pénal et de criminologie, 7, 676-683.

Wacquant, L. (1989). Corps et âme. Notes ethnographiques d'un apprenti boxeur. Actes de la recherche en sciences sociales, 80, 33-67.

Wacquant, L. (1998). La boxe et le blues. In F. Ollier, P. Vassort \& H. Vaugrand (eds), L'illusion sportive, sociologie d'une idéologie totalitaire (223-233). Les cahiers de l'IRSA, nº 2 . 